\title{
Tentativas de Controlo do Peso na População Adulta Portuguesa: Prevalência, Motivos e Comportamentos
}

\author{
Weight Control Attempts among Portuguese Adults: Prevalence, Motives and \\ Behavioral Strategies
}

Inês SANTOS ${ }^{1}$, Ana M. ANDRADE 1 , Pedro J. TEIXEIRA ${ }^{1}$

Acta Med Port 2015 Jan-Feb;28(1):77-86

RESUMO

Introdução: Em Portugal não existem dados atuais sobre tentativas de controlo do peso nem sobre estratégias e motivos na base dessas tentativas na população. Este estudo teve como objectivos determinar a prevalência das tentativas de perda/manutenção do peso e identificar as estratégias comportamentais e os motivos associados, numa amostra representativa da população adulta portuguesa.

Material e Métodos: Estudo transversal constituído por 1098 indivíduos adultos. A informação sociodemográfica, os dados antropométricos e as estratégias e motivos associados à perda/manutenção do peso foram recolhidos por entrevista telefónica.

Resultados: Cerca de $44 \%$ dos adultos portugueses (53\% de mulheres e $35 \%$ dos homens) estão ativamente a tentar controlar o peso. Salienta-se que $22 \%$ das mulheres com peso normal tentam perder peso e que $53 \%$ dos homens e $34 \%$ das mulheres com peso excessivo não o fazem. Entre os homens, $49 \%$ com nível educacional superior estão a tentar controlar o peso versus $32 \%$ com nível educacional básico. A estratégia mais frequentemente adoptada para gerir o peso é o consumo regular de hortícolas e os motivos mais referenciados são melhorar a saúde/prevenir doenças e melhorar o bem-estar.

Discussão e Conclusão: Mais de metade das mulheres e cerca de um terço dos homens em Portugal estão ativamente a tentar gerir o seu peso, utilizando estratégias comportamentais consistentes com as recomendações de saúde pública. Os motivos de saúde e bem-estar predominam sobre os restantes. Este estudo contribui para o conhecimento da gestão do peso em Portugal, alertando para o desenvolvimento de estratégias de prevenção da obesidade adequadas ao perfil da população.

Palavras-chave: Comportamento; Excesso de Peso; Obesidade; Perda de Peso; Portugal.

\section{ABSTRACT}

Introduction: In Portugal, there are no representative data on how many people are actively trying to control their weight and which strategies and motives underlie those attempts. The aim of this study was to estimate the prevalence of weight loss/maintenance attempts and to identify the associated behavioral strategies and motives, in a representative sample of Portuguese adults.

Material and Methods: Cross-sectional study with a sample of 1098 Portuguese adults. Sociodemographic information, anthropometric data and weight loss/maintenance strategies and motives were assessed by telephone interview.

Results: About $44 \%$ of Portuguese adults (53\% women and $35 \%$ men) are actively trying to control their weight. About $22 \%$ of women with normal weight are trying to lose weight while $53 \%$ of men and $34 \%$ of women with excess weight are not trying to manage their weight. About $49 \%$ of men with higher educational level are trying to control their weight, which compares to $32 \%$ among the least educated men. The most frequently used strategy to manage weight is regular vegetable consumption and the motives most frequently reported were improving health/preventing diseases and improving wellbeing.

Discussion and Conclusion: More than half of Portuguese women and about one-third of men are actively trying to control their weight, using behavioral strategies which are generally consistent with public health recommendations. The predominant motives are related to improving health and wellbeing. This study contributes to understanding weight management in Portugal, and could be useful in the development of obesity prevention strategies that match the population profile.

Keywords: Behavior; Obesity; Overweight; Portugal; Weight Loss.

\section{INTRODUÇÃO}

A obesidade apresenta-se como um dos mais importantes desafios de saúde pública nas sociedades afluentes e a sua prevalência tem vindo a aumentar globalmente nas últimas décadas. ${ }^{1}$ Os últimos dados de prevalência publicados em Portugal indicam que cerca de $67 \%$ dos homens e $58 \%$ das mulheres adultas portuguesas têm excesso de peso ou obesidade (Índice de Massa Corporal (IMC) $\geq 25$ $\left.\mathrm{kg} / \mathrm{m}^{2}\right){ }^{2} \mathrm{Na}$ etiologia desta patologia estão, não só a predisposição genética mas também mudanças sociais, económicas, culturais e físicas do meio ambiente. ${ }^{1}$ Está bem descrito na literatura que a obesidade aumenta o risco de diversas doenças crónicas e as guidelines internacionais para a gestão do peso em adultos indicam que alterações do estilo de vida que conduzam à manutenção de $3-5 \%$ do peso perdido acarretam melhorias substanciais nos factores de risco para estas doenças. ${ }^{3}$ No entanto, a maioria das intervenções de perda de peso têm revelado resultados apenas a curto prazo, sendo o peso perdido geralmente recuperado no espaço de três a cinco anos. De facto, a manutenção do peso perdido a longo prazo continua a ser um desafio para muitas pessoas. Por todas estas razões, tentar controlar o peso é hoje parte integrante do estilo de vida de muitos indivíduos. ${ }^{4,5}$

Existem várias orientações no que respeita a estratégias e comportamentos, no contexto da alimentação e da atividade física, que resultam na gestão do peso a longo prazo. Por exemplo, o National Institute for Health and Care Excellence (NICE) ${ }^{6}$ recomenda a ingestão de ali-

1. Centro Interdisciplinar de Estudo da Performance Humana (CIPER). Faculdade de Motricidade Humana. Universidade de Lisboa. Lisboa. Portugal.

Recebido: 07 de Agosto de 2014 - Aceite: 23 de Dezembro de 2014 | Copyright @ Ordem dos Médicos 2015 
mentos ricos em fibra, o consumo de pelo menos 5 porções de uma variedade de frutos e vegetais, a redução da ingestão energética e do consumo de gorduras, evitar o consumo de bebidas alcoólicas e bebidas açucaradas, tomar o pequeno-almoço diariamente, minimizar o tempo sedentário e praticar atividade física diariamente (entre outras). Atualmente, as estratégias mais utilizadas para gerir o peso, documentadas na literatura, são a redução da ingestão energética e o aumento da atividade física, de forma individualizada. ${ }^{7-10}$ Contudo, para além destas estratégias promotoras de saúde, os estudos revelam que por vezes são também utilizadas outras práticas de controlo do peso potencialmente prejudiciais para a saúde (por exemplo, uso de laxantes, diuréticos, suplementos, fazer jejum prolongado). ${ }^{11,12} \mathrm{Na}$ base das tentativas de controlo do peso estão mais frequentemente motivos relacionados com a saúde, sendo a aparência e o bem-estar físico e psicológico outras das razões mais comuns. ${ }^{13,14}$

Em Portugal, esta área de estudo tem sido descurada e não existem, na literatura científica, dados atuais representativos que permitam tirar conclusões sobre o número de indivíduos que está ativamente a tentar controlar o peso, isto é, perder e/ou manter o peso, que estratégias utilizam especificamente para esse efeito, e que motivos estão na base dessas tentativas. Assim, e dado o crescente ênfase na prevenção do aumento de peso ${ }^{3}$, uma melhor compreensão do comportamento dos portugueses relativamente ao controlo do peso, incluindo as estratégias e motivos, será útil para direcionar as políticas de saúde pública e para a promoção de práticas de gestão do peso mais efetivas na população adulta portuguesa. O presente estudo tem como objetivos determinar a prevalência das tentativas de perda e manutenção do peso numa amostra representativa da população adulta portuguesa, e identificar as estratégias comportamentais utilizadas, no âmbito da alimentação e da atividade física, assim como os motivos associados ao controlo do peso.

\section{MATERIAL E MÉTODOS}

A seleção dos participantes foi feita com base numa amostragem probabilística de números telefónicos, na qual foram incluídos indicativos de rede fixa e de rede móvel. Os números telefónicos de rede fixa foram gerados aleatoriamente com estratificação por Região de Saúde, proporcionalmente à população correspondente aos limites geográficos à data. Segundo os resultados do Inquérito às Despesas das Famílias 2010/2011,15 67,7\% dos agregados familiares possuíam telefone fixo e $87,7 \%$ possuíam telemóvel. Desta forma, prevê-se que existam cerca de 1,2 vezes mais números de telemóvel comparativamente com números de rede fixa. Assim, estipulou-se que $83 \%$ das chamadas telefónicas seriam para rede móvel, a distribuir pelos três operadores dominantes de rede móvel nacionais (TMN, Optimus e Vodafone), e 17\% seriam para rede fixa, a distribuir pelos 51 prefixos nacionais. A seleção da amostra e recolha de dados foi realizada por investigadores da Unidade de Epidemiologia do Instituto de Medicina Preven- tiva da Faculdade de Medicina da Universidade de Lisboa, durante o mês de setembro de 2012, através de entrevistas telefónicas. As entrevistas telefónicas foram realizadas em horário laboral nos 5 dias da semana e todos os investigadores receberam formação específica para a recolha de dados.

Os agregados familiares foram selecionados por construção aleatória do número telefónico a partir da aleatorização dos seus dígitos. Visto que com este método há a possibilidade de selecionar números listados e não listados, os potenciais participantes foram contactados a partir das listagens telefónicas previamente elaboradas e foram feitos contactos até se atingir o número de participantes necessário para se assumir a representatividade da população portuguesa (Portugal continental e ilhas) por sexo e grupo etário (18-40 anos; 41-65 anos) - cálculo amostral de 1068 indivíduos.

Dos 1847 indivíduos contactados com sucesso, 363 recusaram participar e 386 foram excluídos por não preencherem os critérios de elegibilidade - residiam em alojamentos colectivos, residiam em Portugal há menos de um ano à data da entrevista ou não tinham nacionalidade portuguesa, não conseguiram responder com acuidade às questões ou eram gestantes e lactantes. Assim, a amostra ficou constituída por 1098 indivíduos elegíveis, isto é, com nacionalidade portuguesa, residência particular e idade compreendida entre os 18 e os 65 anos. Todos os participantes deram consentimento verbal livre, específico e informado antes de participarem no estudo, de acordo com o protocolo aprovado.

Este estudo foi aprovado pela Comissão de Ética para a Saúde do Hospital de Santa Maria. Foi tida em conta a legislação nacional respeitante à proteção e confidencialidade dos dados pessoais (processo $n^{\circ}$ 10026/2012 da Comissão Nacional de Proteção de Dados), pelo que não foi constituída nenhuma base de dados nominal dos participantes.

Para a realização deste estudo, desenvolveu-se um questionário específico (Appendix) com base em instrumentos psicométricos recomendados na literatura, que incluiu 11 questões sociodemográficas, englobando idade, região de residência, estado civil, nível educacional e nível socio-económico (calculado através do índice de Graffar), ${ }^{16}$ 12 questões relativas às tentativas de perda e manutenção do peso, incluindo estratégias e motivos associados, e ainda 8 questões relativas à história do peso, incluindo peso e altura à data da entrevista, entre outras (documento suplementar). Com base no peso e na altura auto-reportados, calculámos o IMC $\left(\mathrm{kg} / \mathrm{m}^{2}\right)$, o qual foi categorizado, de acordo com o preconizado pela Organização Mundial de Saúde, ${ }^{17} \mathrm{em}$ : peso normal (IMC $\geq 18,5 \leq 25 \mathrm{~kg} / \mathrm{m}^{2}$ ), pré-obesidade (IMC $\geq 25$ e $<30 \mathrm{~kg} / \mathrm{m}^{2}$ ) e obesidade (IMC $\geq 30$ $\left.\mathrm{kg} / \mathrm{m}^{2}\right)$.

\section{Tentativas de controlo do peso}

De forma a avaliar as tentativas de controlo do peso, os participantes indicaram o que tinham tentado fazer nos 
últimos 12 meses relativamente ao seu peso, com base em 7 categorias de resposta: 1) perder algum peso; 2) manter o seu peso, para não aumentar; 3) manter o seu peso, para não perder; 4) aumentar de peso; 5) nada, não se preocupou de todo com o seu peso; 6) nada, mas há mais de 12 meses, e já depois de completar 18 anos, procurou perder ou manter o peso; 7) não sabe/não responde. Para o propósito deste estudo, as categorias 3) e 4) e as categorias 5) e 6) foram agrupadas.

\section{Estratégias comportamentais}

Aos participantes que referiram estar a tentar perder algum peso ou manter o peso para não aumentar foi-Ihes pedido que indicassem, numa escala dicotómica (sim ou não), as estratégias (de uma lista de 18) que estavam a utilizar ou tinham utilizado no passado para tentar gerir o peso (por exemplo, 'para controlar o seu peso pratica atividade física de forma regular, isto é, pelo menos 3 vezes por semana').

\section{Motivos}

Relativamente aos motivos para tentar perder ou manter o peso, os participantes responderam numa escala de Likert de 4 pontos (de 'discordo fortemente' a 'concordo fortemente') a uma lista de 8 razões que os levaram a tentar controlar o peso (por exemplo, 'para melhorar a saúde em geral ou prevenir doenças futuras').

\section{Análise estatística}

Para a análise estatística dos dados utilizámos o programa IBM Statistical Package for the Social Sciences ${ }^{\circledR}$ (SPSS), versão 22. Os resultados estão apresentados em percentagem. Utilizou-se o teste qui-quadrado para comparação entre grupos. Os dados estão expressos com intervalos de confiança de 95\% (nível de significância de $\alpha=0,05$ ).

\section{RESULTADOS}

No total, $24,3 \%$ ( $n=267$ ) dos adultos portugueses reportaram estar a tentar perder peso, $19,4 \%(n=213)$ a tentar manter o peso (isto é, evitar aumentar de peso) e $6,5 \%$ $(n=71)$ a tentar aumentar de peso ou manter o peso para não perder (isto é, evitar perder peso), sendo que 49,8\% $(n=547)$ reportaram não estar a fazer nada relativamente ao seu peso.

Considerando as tentativas de controlo do peso por sexo (Fig. 1), constata-se que mais de metade das muIheres adultas portuguesas estão a tentar controlar o peso (perder peso ou evitar aumentar), ao passo que nos homens esta prevalência foi de cerca de um terço.

Mais de metade dos adultos portugueses que reportaram ter pré-obesidade ou obesidade afirmaram estar a tentar controlar o peso (Fig. 2). Ainda assim, cerca de 44\% indicaram não ter qualquer intenção de controlar o peso. No que respeita aos indivíduos que reportaram ter um peso dentro dos parâmetros da normalidade, apesar de a maioria reportar não estar a tentar controlar o peso, cerca de $39 \%$ afirmaram ter essa intenção.

A Tabela 1 apresenta a prevalência das tentativas de controlo do peso de acordo com as características sociodemográficas. Não se observaram diferenças significativas entre os grupos de controlo do peso no que respeita à idade, estado civil e nível socioeconómico $(p>0,05)$. Nas mulheres, mas não nos homens, observaram-se diferenças significativas relativamente à região de residência: uma maior proporção de mulheres residentes na zona de Lisboa e Vale do Tejo e na Madeira estavam a tentar perder peso, ao passo que na região do Alentejo mais mulheres estavam a tentar manter o peso; nas restantes regiões houve mais mulheres a reportarem não estar a tentar controlar o peso $(p<0,001)$. Nos homens observaram-se diferenças

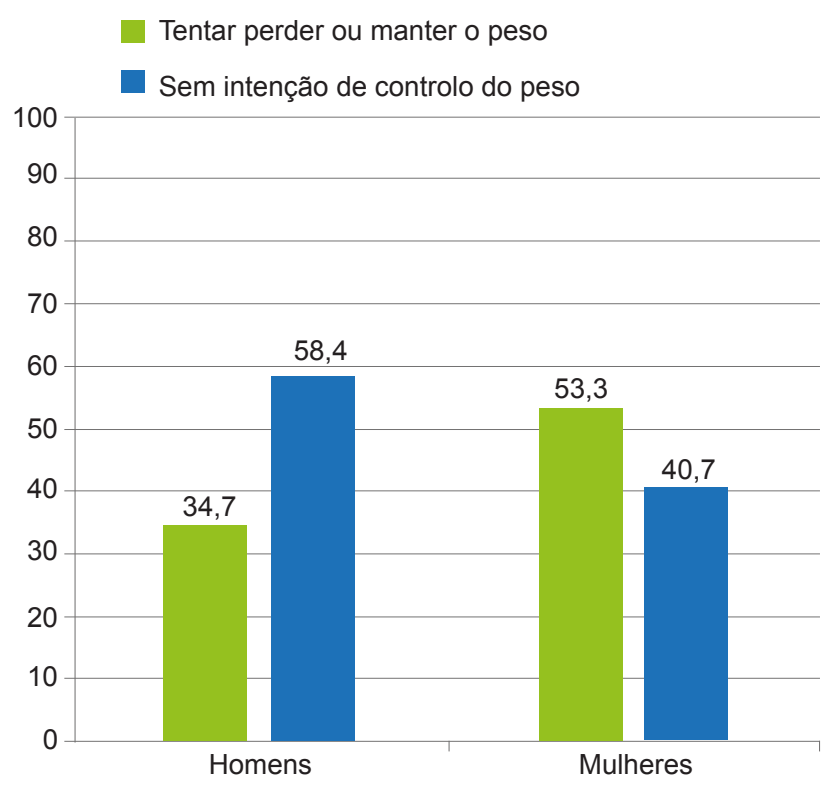

Figura 1 - Prevalência das tentativas de controlo do peso, por sexo, na população adulta portuguesa. Valores expressos em percentagem.

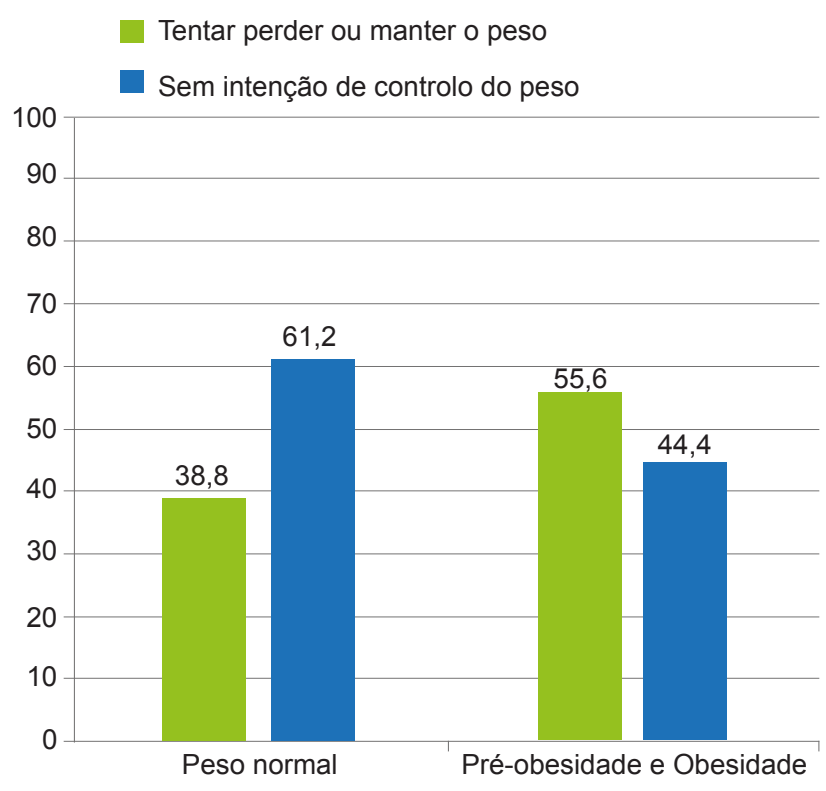

Figura 2 - Prevalência das tentativas de controlo do peso, por categoria ponderal, na população adulta portuguesa. Valores expressos em percentagem. 
Tabela 1 - Prevalência das tentativas de controlo do peso de acordo com as características sociodemográficas (homens)

\begin{tabular}{|c|c|c|c|c|}
\hline \multirow[b]{2}{*}{ Características } & \multicolumn{4}{|c|}{ Homens $(n=565)$} \\
\hline & Tentar perder peso & Tentar manter o peso & Sem intenção de controlo do peso & $\boldsymbol{p}^{*}$ \\
\hline Idade & & & & 0,179 \\
\hline $18-40$ anos & 22,3 & 17,1 & 60,6 & \\
\hline 41-65 anos & 16,0 & 19,1 & 65,0 & \\
\hline Região de residência & & & & 0,958 \\
\hline Norte & 20,8 & 20,3 & 58,9 & \\
\hline Centro & 17,9 & 15,2 & 67,0 & \\
\hline Lisboa e Vale do Tejo & 18,6 & 16,0 & 65,4 & \\
\hline Alentejo & 12,5 & 25,0 & 62,5 & \\
\hline Algarve & 23,5 & 17,6 & 58,8 & \\
\hline Madeira & 25,0 & 12,5 & 62,5 & \\
\hline Açores & 18,8 & 25,0 & 56,3 & \\
\hline Estado civil & & & & 0,144 \\
\hline Solteiro & 25,4 & 15,4 & 59,2 & \\
\hline Casado/União de facto & 15,9 & 19,9 & 64,2 & \\
\hline Divorciado/Viúvo & 18,9 & 17,0 & 64,2 & \\
\hline Nível educacional & & & & 0,009 \\
\hline Ensino básico & 16,7 & 15,3 & 68,1 & \\
\hline Ensino secundário & 20,5 & 14,1 & 65,4 & \\
\hline Ensino superior & 22,2 & 26,4 & 51,4 & \\
\hline Nível Socioeconómico & & & & 0,145 \\
\hline Elevado & 20,4 & 23,4 & 56,2 & \\
\hline Médio & 17,6 & 14,5 & 67,9 & \\
\hline Baixo & 22,2 & 13,9 & 63,9 & \\
\hline Índice de Massa Corporal & & & & $<0,001$ \\
\hline Peso normal & 9,8 & 17,3 & 72,8 & \\
\hline Pré-obesidade & 23,5 & 21,7 & 54,8 & \\
\hline Obesidade & 46,3 & 7,4 & 46,3 & \\
\hline
\end{tabular}

Valores expressos em percentagem.

* Teste qui-quadrado para comparação entre grupos (diferenças estatisticamente significativas assinaladas a negrito).

significativas no que diz respeito ao nível educacional: apesar da maioria reportar não estar a tentar controlar o peso, mais homens com ensino básico e secundário reportaram estar a tentar perder peso e mais homens com ensino superior reportaram estar a tentar manter o peso $(p=0,009)$. Como esperado, uma maior proporção de mulheres com pré-obesidade e obesidade reportaram estar a tentar perder peso, ao passo que mais mulheres com peso normal reportaram estar a tentar manter o peso ou a não fazer nada relativamente ao seu peso $(p<0,001)$. Em contraste, a mesma proporção de homens com obesidade reportou estar a tentar perder peso e não ter qualquer intenção de controlo do peso, e mais homens com peso normal e com pré-obesidade reportaram não estar a tentar controlar o peso $(p<0,001)$.

A estratégia comportamental mais frequentemente adoptada pela população adulta portuguesa, tanto com vista à perda de peso como à manutenção do peso, foi o consumo regular de produtos hortícolas nas refeições principais (Tabela 2). O consumo regular de sopa nas refeições principais, a ingestão de água em detrimento de outras bebidas, o consumo regular de pequeno-almoço, a inclusão de pequenas merendas a meio da manhã e da tarde, optar por consumir pequenas porções, praticar atividade física regularmente, procurar informar-se sobre nutrição e atividade física e selecionar de forma consciente os alimentos foram outras das estratégias utilizadas por mais de metade da população portuguesa (com vista a gerir o peso). Algumas 
Tabela 1 - Prevalência das tentativas de controlo do peso de acordo com as características sociodemográficas (mulheres)

\begin{tabular}{|c|c|c|c|c|}
\hline \multirow[b]{2}{*}{ Características } & \multicolumn{3}{|c|}{ Mulheres $(n=533)$} & \multirow[b]{2}{*}{$p^{*}$} \\
\hline & Tentar perder peso & Tentar manter o peso & Sem intenção de controlo do peso & \\
\hline Idade & & & & 0,206 \\
\hline $18-40$ anos & 29,3 & 25,5 & 45,2 & \\
\hline 41-65 anos & 36,6 & 21,8 & 41,6 & \\
\hline Região de residência & & & & $<0,001$ \\
\hline Norte & 25,8 & 26,3 & 47,8 & \\
\hline Centro & 26,0 & 27,6 & 46,3 & \\
\hline Lisboa e Vale do Tejo & 49,2 & 13,3 & 37,5 & \\
\hline Alentejo & 30,8 & 46,2 & 23,1 & \\
\hline Algarve & 39,3 & 14,3 & 46,4 & \\
\hline Madeira & 71,4 & 14,3 & 14,3 & \\
\hline Açores & 21,4 & 35,7 & 42,9 & \\
\hline Estado civil & & & & 0,190 \\
\hline Solteiro & 27,3 & 30,2 & 42,4 & \\
\hline Casado/União de facto & 35,7 & 20,4 & 43,9 & \\
\hline Divorciado/Viúvo & 35,4 & 21,5 & 43,1 & \\
\hline Nível educacional & & & & 0,097 \\
\hline Ensino básico & 32,6 & 18,6 & 48,8 & \\
\hline Ensino secundário & 36,2 & 24,1 & 39,7 & \\
\hline Ensino superior & 31,3 & 29,9 & 38,9 & \\
\hline Nível Socioeconómico & & & & 0,249 \\
\hline Elevado & 36,3 & 26,9 & 36,8 & \\
\hline Médio & 29,8 & 22,7 & 47,5 & \\
\hline Baixo & 39,3 & 14,3 & 46,4 & \\
\hline Índice de Massa Corporal & & & & $<0,001$ \\
\hline Peso normal & 22,1 & 27,0 & 50,9 & \\
\hline Pré-obesidade & 44,4 & 20,5 & 35,1 & \\
\hline Obesidade & 56,3 & 14,1 & 29,7 & \\
\hline
\end{tabular}

Valores expressos em percentagem.

* Teste qui-quadrado para comparação entre grupos (diferenças estatisticamente significativas assinaladas a negrito).

estratégias foram significativamente mais prevalentes nas mulheres a tentar perder peso, comparativamente com as mulheres a tentar manter o peso, nomeadamente a ingestão regular de pequeno-almoço $(p=0,042)$, receber orientação de um especialista $(p=0,001)$, tomar medicamentos ou suplementos $(p<0,001)$, frequentar um programa de controlo do peso $(p=0,001)$, tomar laxantes ou diuréticos $(p=0,001)$ e induzir o vómito ou fazer jejum prolongado $(p=0,016)$.

Comparando as estratégias utilizadas para controlar o peso por sexo, apenas em seis das estratégias não se observaram diferenças significativas; uma maior proporção de homens referiu praticar atividade física de forma regular $(p=0,004)$ e uma maior proporção de mulheres referiu beber água em detrimento de outras bebidas $(p<0,001)$, consumir pequenas porções $(p<0,001)$, incluir pequenas merendas a meio da manhã e da tarde $(p=0,001)$, selecionar de forma consciente os alimentos $(p<0,001)$, tomar o pequeno-almoço regularmente $(p=0,011)$, receber orientação de um especialista $(p=0,002)$, contabilizar as calorias das refeições $(p=0,019)$, tomar medicamentos ou suplementos $(p<0,001)$, tomar laxantes ou diuréticos $(p=0,001)$, frequentar um programa de controlo do peso $(p=0,009)$ e fazer dietas 'da moda' $(p=0,016)$.

Os motivos mais frequentemente reportados pela população adulta portuguesa para controlar o peso foram melhorar a saúde em geral ou prevenir doenças futuras e melhorar o bem-estar no dia-a-dia (> 90\%) (Tabela 3). 
Tabela 2 - Estratégias comportamentais utilizadas para controlar o peso, por intenção de controlo do peso (homens)

\begin{tabular}{|c|c|c|c|}
\hline \multirow[b]{2}{*}{ Estratégias } & \multicolumn{3}{|c|}{ Homens $(n=196)$} \\
\hline & Tentar perder peso & Tentar manter o peso & Total \\
\hline Consumir regularmente hortícolas nas refeições principais & 42,6 & 41,0 & 83,6 \\
\hline Consumir regularmente sopa nas refeições principais & 36,4 & 33,8 & 70,3 \\
\hline Beber água em detrimento de outras bebidas & 34,9 & 31,3 & 66,2 \\
\hline Tomar o pequeno-almoço regularmente & 31,1 & 29,0 & 60,1 \\
\hline Consumir pequenas merendas a meio da manhã/tarde & 32,1 & 27,6 & 59,7 \\
\hline Comer pequenas porções & 33,2 & 26,4 & 59,6 \\
\hline Praticar atividade física de forma regular & 28,9 & 26,8 & 55,7 \\
\hline Procurar informar-se sobre nutrição/atividade física & 29,0 & 25,9 & 54,9 \\
\hline Selecionar de forma consciente os alimentos & 27,7 & 26,7 & 54,4 \\
\hline Comer pausadamente e mastigar bem os alimentos & 23,2 & 23,7 & 46,9 \\
\hline Receber orientação de um especialista & 10,2 & 6,6 & 16,8 \\
\hline Contabilizar as calorias das refeições & 6,1 & 3,1 & 9,2 \\
\hline Fazer registos alimentares/atividade física & 3,1 & 4,1 & 7,1 \\
\hline Tomar medicamentos/suplementos & 3,6 & 1,0 & 4,6 \\
\hline Frequentar um programa de controlo do peso & 2,6 & 1,5 & 4,1 \\
\hline Tomar laxantes/diuréticos & 2,6 & 1,0 & 3,6 \\
\hline Induzir o vómito/jejum prolongado & 2,6 & 0,5 & 3,1 \\
\hline Fazer dietas "da moda" & 0,5 & 1,0 & 1,5 \\
\hline
\end{tabular}

Uma maior proporção de homens referiu tentar controlar o peso para melhorar a condição física ou conseguir praticar desporto mais facilmente $(p=0,002)$ e para cumprir requisitos específicos da sua profissão ou ocupação $(p=0,040)$, comparativamente com as mulheres. Pelo contrário, uma maior proporção de mulheres referiu tentar controlar o peso por motivos relacionados com manter ou obter uma aparência física confortável $(p=0,001)$, melhorar a autoestima em relação ao seu corpo $(p<0,001)$ e evitar ser discriminada ou para se sentir mais bem integrada na sociedade $(p=0,009)$ em comparação com os homens. Observaram-se ainda diferenças significativas nos motivos para tentar perder versus manter o peso em ambos os sexos: uma maior proporção de homens a tentar manter o peso referiu fazê-lo para cumprir requisitos específicos da sua profissão ou ocupação $(p=0,007)$ e para evitar ser descriminado ou para se sentir mais bem integrado na sociedade ( $p=0,049)$, comparativamente com os homens a tentar perder peso; e uma maior proporção de mulheres a tentar perder peso referiu fazê-lo para melhorar o bem-estar no dia-a-dia $(p=0,023)$ e para melhorar a autoestima em relação ao corpo $(p=0,043)$, comparativamente com as mulheres na fase de manutenção.

\section{DISCUSSÃO}

O presente estudo revelou que cerca de $44 \%$ dos adultos portugueses estão ativamente a tentar controlar o peso, mais especificamente cerca de $24 \%$ estão a tentar perder peso e cerca de $19 \%$ estão a tentar manter o peso, sendo a prevalência de ambos os indicadores superior nas muIheres. Da revisão efectuada, apenas um estudo com uma amostra representativa da população adulta portuguesa retratou esta temática, tendo concluído que cerca de $16 \%$ dos adultos portugueses estavam a tentar controlar o peso em 2001. ${ }^{18}$ Este aumento nas tentativas de controlo do peso reflete as tendências relativas à prevalência de obesidade na população adulta portuguesa, ${ }^{2}$ facto que também parece verificar-se noutras populações. Por exemplo, nos EUA, dados do Behavioral Risk Factor Surveillance System revelaram que a proporção de indivíduos adultos a tentar perder peso era de 32,3\% em 1989 e de 37,3\% em 1999 19,20 e dados do National Health and Nutrition Examination Survey mostraram que a prevalência de tentativas de perda de peso era de $32,9 \%$ no período de $1988-1994$ e de $48 \%$ entre 2003-2008..$^{8,21}$ Para além do aumento da obesidade per se, outros fatores podem também ajudar a perceber a tendência de crescimento nas tentativas de gerir o peso, 
Tabela 2 - Estratégias comportamentais utilizadas para controlar o peso, por intenção de controlo do peso (mulheres)

Mulheres $(n=284)$

Estratégias

Tentar perder peso Tentar manter o peso ${ }^{\mp \mp} \quad$ Total $^{\mp}$

Consumir regularmente hortícolas nas refeições principais

\begin{tabular}{lll}
51,1 & 37,2 & $88,3^{1}$ \\
41,7 & 31,8 & $73,5^{5}$ \\
47,3 & 36,7 & $84,1^{2 * * *}$ \\
39,0 & $32,3^{*}$ & $71,3^{7 *}$ \\
41,7 & 32,9 & $74,6^{4 * *}$ \\
43,2 & 32,5 & $75,7^{3 * * *}$ \\
23,4 & 18,8 & $42,2^{10 * *}$ \\
34,8 & 25,9 & $60,6^{8}$ \\
40,4 & 31,9 & $72,3^{6 * * *}$ \\
29,1 & 22,0 & $51,1^{9}$ \\
21,3 & $7,8^{* *}$ & $29,1^{11 * *}$ \\
11,3 & 5,3 & $16,7^{12 *}$ \\
6,4 & 2,5 & $8,8^{16}$ \\
15,6 & $1,1^{* * *}$ & $16,7^{13 * * *}$ \\
9,2 & $1,4^{* *}$ & $10,6^{15 * *}$ \\
10,3 & $1,8^{* *}$ & $12,1^{14 * *}$ \\
3,9 & $0,4^{*}$ & $4,3^{18}$ \\
4,6 & 1,4 & $6,0^{17 *}$ \\
\hline
\end{tabular}

Fazer dietas "da moda"

Pese embora sejam bem reconhecidos os benefícios como alterações na norma social face à obesidade e os seus riscos, um aumento do número de programas e produtos visando a gestão de peso (comerciais, nos serviços de saúde, etc.), ou uma maior importância atribuída pela população ao peso ou forma do corpo, à saúde em geral, ou à relação entre os dois.

Considerando as tentativas de controlo do peso por categoria de IMC, é de salientar a proporção de mulheres com peso normal a tentar perder peso $(22,1 \%)$. Yaemsiri e colaboradores ${ }^{8}$ observaram que cerca de $29 \%$ das muIheres americanas adultas com peso saudável percepcionavam-se como tendo peso excessivo, sendo esta auto-percepção um forte preditor das tentativas de perda de peso. Em Portugal, Santos et $\mathrm{al}^{18}$ constataram que cerca de $52 \%$ das mulheres adultas com peso normal sentiam ter peso excessivo. Este aspeto não foi avaliado no presente estudo, sendo por isso necessário mais dados para determinar em que medida as tentativas de controlo do peso nas mulheres portuguesas com peso normal são resultado de uma percepção distorcida do que é um peso saudável ou o reflexo de uma imagem corporal empobrecida que as leva a tentar alcançar o ideal de magreza valorizado socialmente (ou uma combinação de ambos os fatores).

para a saúde da perda de peso estável e duradoura, ${ }^{3}$ é notável que mais de metade dos homens $(53,1 \%)$ e $33,5 \%$ das mulheres portuguesas com peso excessivo (nota: $46,3 \%$ dos homens e $29,7 \%$ das mulheres com obesidade; dados não reportados) não estejam a tentar controlar o peso. Por um lado, a evidência científica revela que o sucesso da gestão do peso a longo prazo é modesto e que a experiência de repetidas tentativas de controlo do peso mal sucedidas podem conduzir ao desenvolvimento de processos cognitivos de descrença aprendida (por internalização do insucesso) e, consequentemente, a atitudes de cepticismo e passividade. ${ }^{4,22,23}$ Por outro lado, podem também ocorrer muitos casos em que não existe suficiente consciência do que é um peso saudável (face ao próprio peso) e das vantagens em tentar obtê-lo. Estes são os casos para os quais se salienta o papel dos clínicos e das autoridades de saúde ao promover a perda e a manutenção do peso a longo prazo na população. Finalmente, são também possíveis casos em que as pessoas tomaram uma decisão consciente e informada no sentido de não procurarem a perda de peso, possivelmente por valorizarem mais outros aspetos da sua vida e da gestão da sua saúde. Estudos futuros deverão 
Tabela 3 - Motivos para controlar o peso, por intenção de controlo do peso (homens)

\begin{tabular}{|c|c|c|c|}
\hline \multirow[b]{2}{*}{ Motivos } & \multicolumn{3}{|c|}{ Homens $(n=196)$} \\
\hline & Tentar perder peso & Tentar manter o peso ${ }^{\mp \mp}$ & Total \\
\hline Melhorar saúde em geral/prevenir doenças futuras & 49,0 & 47,4 & 96,4 \\
\hline Melhorar o bem-estar no dia-a-dia & 49,0 & 47,4 & 96,4 \\
\hline Melhorar a condição física/conseguir praticar desporto & 43,8 & 41,2 & 85,1 \\
\hline Manter/obter uma aparência física confortável & 43,1 & 41,0 & 84,1 \\
\hline Melhorar a autoestima em relação ao corpo & 41,3 & 39,3 & 80,6 \\
\hline Resolver problema de saúde/indicação de um profissional & 32,5 & 29,9 & 62,4 \\
\hline Cumprir requisitos específicos da profissão & 17,3 & $25,5^{\star *}$ & 42,9 \\
\hline Evitar ser descriminado/integrar-se melhor na sociedade & 14,3 & $19,9^{*}$ & 34,2 \\
\hline
\end{tabular}

Valores expressos em percentagem. Os valores correspondem às respostas 'concordo' e 'concordo totalmente' para cada motivo.

${ }^{*} p<0,05 ;{ }^{* *} p<0,01$ (Teste qui-quadrado para comparação entre grupos). ${ }^{\mp}$ Diferenças entre sexos. ${ }^{\mp \mp}$ Diferenças entre tentar perder peso e tentar manter o peso.

ajudar a identificar e distinguir as razões para a não procura da perda de peso em pessoas com obesidade.

Os resultados do presente estudo revelaram também que cerca de $49 \%$ dos homens portugueses com nível educacional superior estão ativamente a tentar controlar o peso (comparando com $32 \%$ e $35 \%$ dos homens com nível educacional básico e secundário, respectivamente). O nível educacional tem sido consistentemente associado às tentativas de controlo do peso em estudos populacionais, assim como a adesão às estratégias de saúde pública recomendadas para controlar o peso, com prevalências aumentadas à medida que aumentam os anos de escolaridade. ${ }^{11,24}$ Uma tendência associativa oposta tem sido encontrada para a prevalência de obesidade em adultos, ${ }^{25,26}$ incluindo na população portuguesa. ${ }^{2,27} \mathrm{Ou}$ seja, a obesidade é marcadamente mais prevalente em pessoas de nível educacional (e socioeconómico) mais baixo. De facto, dados do presente estudo revelam que cerca de $47 \%$ e $64 \%$ dos indivíduos portugueses com pré-obesidade e obesidade, respectivamente, têm um nível educacional básico, sendo que apenas $23 \%$ e $12 \%$ destes mesmos indivíduos apresentam um nível educacional superior (dados não reportados). O nível educacional potencia não só um rendimento económico mais elevado, que permite uma maior aquisição de bens e serviços de saúde, mas também as competências cognitivas, assim como o acesso a informação relacionada com a saúde, os quais podem influenciar os comportamentos de saúde, incluindo os que afectam diretamente o peso corporal. ${ }^{25}$ Reforça-se, por isso, a importância do desenvolvimento de estratégias de promoção do controlo do peso considerando as desigualdades ao nível da literacia da população portuguesa.

Os adultos portugueses que revelaram estar a tentar controlar o peso afirmaram fazê-lo recorrendo a várias estratégias do foro comportamental. É encorajador observar que as estratégias mais frequentemente utilizadas pela po- pulação adulta portuguesa para tentar controlar o peso vão de encontro às recomendações da Organização Mundial de Saúde, priorizadas na política da Carta Europeia de Luta Contra a Obesidade. ${ }^{1} \mathrm{O}$ consumo de hortícolas e sopa com regularidade, a água como bebida de eleição, a inclusão diária de pequeno-almoço e merendas nos intervalos das refeições principais, a prática regular de atividade física e a literacia alimentar e nutricional são estratégias integrantes do estilo de vida saudável promovido pelo Programa Nacional para a Promoção da Alimentação Saudável da Direção-Geral da Saúde. ${ }^{28}$ É importante notar que as estratégias adoptadas com o intuito de perder e manter o peso foram sensivelmente as mesmas, tanto nos homens como nas mulheres, com exceção de seis estratégias consideradas especialmente úteis pelas mulheres na fase de perda de peso. Três destas estratégias - tomar suplementos alimentares para emagrecer (provavelmente sem recomendação específica), tomar laxantes ou diuréticos e induzir o vómito ou fazer jejum prolongado - e ainda, fazer dietas "da moda" (que usualmente são nutricionalmente desequilibradas) são estratégias potencialmente perigosas para a saúde, principalmente se mantidas a longo prazo. Apesar de terem sido adoptadas por uma pequena proporção de homens e mulheres, o que é consistente com outros estudos epidemiológicos, ${ }^{11,19,29,30}$ devem ser alvo de atenção por parte das entidades promotoras de saúde pois este tipo de estratégias tem sido associado a comportamentos de sobrealimentação, compulsão alimentar e a insucesso no que respeita ao controlo do peso. ${ }^{31-33}$

Observaram-se diferenças específicas por sexo no que respeita à adopção de algumas estratégias para controlar o peso. Em contraste com outros estudos ${ }^{11,20,30} \mathrm{em}$ que a mesma proporção de homens e mulheres reportou praticar atividade física para tentar perder peso, em Portugal a prática de a atividade física é mais prevalente nos homens a tentar gerir o peso. Por outro lado, as mulheres 
Tabela 3 - Motivos para controlar o peso, por sexo e intenção de controlo do peso (mulheres)

Mulheres $(n=284)$

\begin{tabular}{llll} 
Motivos & Tentar perder peso & Tentar manter o peso ${ }^{\mp \mp}$ & Total $^{\mp}$ \\
\cline { 2 - 4 } Melhorar saúde em geral/prevenir doenças futuras & 54,3 & 40,4 & $94,7^{1}$ \\
Melhorar o bem-estar no dia-a-dia & 53,9 & $40,8^{*}$ & $94,7^{2}$ \\
Melhorar a condição física/conseguir praticar desporto & 40,6 & 32,4 & $73,0^{5 * *}$ \\
Manter/obter uma aparência física confortável & 53,5 & 40,1 & $93,6^{3 * *}$ \\
Melhorar a autoestima em relação ao corpo & 52,8 & $40,1^{*}$ & $92,9^{4 * * *}$ \\
Resolver problema de saúde/indicação de um profissional & 37,3 & 28,7 & $65,9^{6}$ \\
Cumprir requisitos específicos da profissão & 18,8 & 14,8 & $33,6^{8 *}$ \\
Evitar ser descriminado/integrar-se melhor na sociedade & 26,2 & 20,1 & $46,2^{7 * *}$
\end{tabular}

Valores expressos em percentagem. Os valores correspondem às respostas 'concordo' e 'concordo totalmente' para cada motivo. Os números sobrescritos correspondem à ordem descendente.

${ }^{*} p<0,05 ;{ }^{* *} p<0,01 ;{ }^{* * *} p<0,001$ (Teste qui-quadrado). ${ }^{\mp}$ Diferenças entre sexos. ${ }^{\mp \mp}$ Diferenças entre tentar perder peso e tentar manter o peso.

reportaram mais frequentemente a utilização de estratégias relacionadas com o comportamento alimentar (por exemplo, comer pequenas porções) e algumas potencialmente nefastas para a saúde (por exemplo, toma de suplementos), estando em concordância com outros estudos. ${ }^{11,19} \mathrm{O}$ conhecimento das estratégias escolhidas por homens e mulheres para tentar perder e manter o peso pode ser útil no delineamento de intervenções promotoras de comportamentos e estilos de vida saudáveis com vista ao controlo do peso nestes dois grupos populacionais em particular.

A mensagem de que a perda de peso tem um papel benéfico na saúde, prevenção de doenças e bem-estar geral parece ter sido bem aceite pela população portuguesa, visto serem as razões mais apontadas para tentar controlar o peso. Estudos internacionais apontam no mesmo sentido, tendo revelado que os motivos mais frequentemente citados pelos indivíduos para tentar perder peso é a saúde e o bem-estar. ${ }^{14,30}$ Talvez menos influenciados pela desejabilidade social do que os motivos de saúde, é de notar que motivos para controlar o peso relacionados com manter ou obter uma boa aparência física e melhorar a autoestima em relação ao corpo apresentam também valores muito elevados. Juntamente com a vontade de evitar ser discriminado ou sentir-se melhor integrado na sociedade, estes foram mais vezes referenciados pelas mulheres, tendo os homens mencionado mais frequentemente motivos relacionados com a melhoria da condição física ou para conseguir praticar desporto e cumprir requisitos específicos da profissão. Apesar destas diferenças significativas entre sexos, e de as mulheres estarem normalmente mais sujeitas a pressões culturais no que respeita ao corpo e ao controlo do peso, ${ }^{34}$ cerca de $80 \%$ dos homens portugueses que estão a tentar perder ou manter o peso fazem-no por questões relacionadas com a aparência e a autoestima em relação ao corpo, facto que também se verifica noutros estudos. ${ }^{30}$
Os resultados deste estudo devem ser interpretados considerando que as medidas utilizadas foram auto-reportadas. O viés associado à desejabilidade social ${ }^{35}$ pode aumentar a indicação da utilização de estratégias comportamentais mais saudáveis para tentarem controlar o peso (por exemplo, prática de atividade física) e reduzir a indicação da utilização de estratégias menos saudáveis (por exemplo, indução do vómito ou jejum prolongado) por parte de alguns indivíduos. $\mathrm{O}$ autorrelato do peso e altura também pode levantar questões relativamente à acurácia dos valores de IMC. Os homens tendem a sobre-reportar o peso e a altura, enquanto que as mulheres tendem a sub-reportar o peso e a sobre-reportar a altura, ${ }^{36,37}$ estatísticas que são ainda mais pronunciadas quando os dados são recolhidos por entrevista telefónica. ${ }^{38}$ Assim, o autorrelato destas medidas pode levar a uma sub- ou sobre-estimação da prevalência de peso normal, pré-obesidade e obesidade. Contudo, alguns estudos sugerem que o peso auto-reportado se correlaciona de forma elevada com o peso medido objectivamente ${ }^{39}$. Outra limitação deste estudo é o facto de não avaliar a eficácia das estratégias comportamentais utilizadas, o que deve ser considerado em estudos futuros. Apesar destas limitações, este estudo destaca-se por ser constituído por uma amostra representativa nacional, permitindo desta forma a generalização dos resultados para a população adulta portuguesa.

\section{CONCLUSÕES}

Mais de um terço dos adultos portugueses estão ativamente a tentar controlar o peso, sendo que a maioria reporta utilizar estratégias comportamentais consistentes com as recomendações de saúde pública. Foram identificadas estratégias comportamentais, no âmbito da alimentação e da atividade física, e motivos específicos por sexo. Esta investigação contribui, assim, para a compreensão dos 
processos associados ao fenómeno da gestão do peso em Portugal, alertando para o desenvolvimento de estratégias de prevenção da obesidade apropriadas à população nacional.

\section{AGRADECIMENTOS}

Os autores agradecem à Unidade de Epidemiologia do Instituto de Medicina Preventiva da Faculdade de Medicina da Universidade de Lisboa, em especial a Violeta Alarcão, pela colaboração no processo de planeamento e recolha

\section{REFERÊNCIAS}

1. World Health Organization European Charter on counteracting obesity. WHO European Ministerial Conference on Counteracting Obesity: Diet and physical activity for health. Istanbul: WHO; 2006.

2. Sardinha LB, Santos DA, Silva AM, Coelho-e-Silva MJ, Raimundo AM Moreira $\mathrm{H}$, et al. Prevalence of overweight, obesity, and abdominal obesity in a representative sample of Portuguese adults. PLoS One. 2012;7:e47883.

3. Jensen MD, Ryan DH, Apovian CM, Ard JD, Comuzzie AG, Donato KA, et al. $2013 \mathrm{AHA} / \mathrm{ACC} / \mathrm{TOS}$ Guideline for the Management of Overweight and Obesity in Adults: A Report of the American College of Cardiology/ American Heart Association Task Force on Practice Guidelines and The Obesity Society. J Am Coll Cardiol. 2014;63:2985-3023.

4. Powell LH, Calvin JE. Effective obesity treatments. Am Psychol. 2007;62:234-46.

5. Wadden TA, Phelan S. Behavioral assessment of the obese patient. In Wadden TA, Stunkard AJ, editors. Handbook of obesity treatment. New York: Guilford Press; 2002. p. 186-226.

6. National Institute for Health and Care Excellence (NICE). Obesity: the prevention, identification, assessment and management of overweight and obesity in adults and children. London: NICE; 2006 [consultado 2014 Dez 3]; Disponível em: http://www.nice.org.uk/guidance/cg43.

7. Andreyeva T, Long MW, Henderson KE, Grode GM. Trying to lose weight: diet strategies among Americans with overweight or obesity in 1996 and 2003. J Am Diet Assoc. 2010;110:535-42.

8. Yaemsiri S, Slining MM, Agarwal SK. Perceived weight status, overweight diagnosis, and weight control among US adults: the NHANES 20032008 Study. Int J Obes. 2011;35:1063-70.

9. Korkeila M, Rissanen A, Kaprio J, Sorensen TI, Koskenvuo M. Weightloss attempts and risk of major weight gain: a prospective study in Finnish adults. Am J Clin Nutr. 1999;70:965-75.

10. Jackson SE, Wardle J, Johnson F, Finer N, Beeken RJ. The impact of a health professional recommendation on weight loss attempts in overweight and obese British adults: a cross-sectional analysis. BMJ Open. 2013;3:e003693.

11. Kruger J, Galuska DA, Serdula MK, Jones DA. Attempting to lose weight: specific practices among U.S. adults. Am J Prev Med. 2004;26:402-6.

12. Williams L, Germov J, Young A. Preventing weight gain: a population cohort study of the nature and effectiveness of mid-age women's weight control practices. Int J Obes. 2007;31:978-86.

13. O'Brien K, Venn BJ, Perry T, Green TJ, Aitken W, Bradshaw A, et al. Reasons for wanting to lose weight: different strokes for different folks. Eat Behav. 2007;8:132-5.

14. Hankey CR, Leslie WS, Lean ME. Why lose weight? Reasons for seeking weight loss by overweight but otherwise healthy men. Int $\mathrm{J}$ Obes Relat Metab Disord. 2002;26:880-2

15. Instituto Nacional de Estatística IP. Inquérito às despesas das famílias 2010/2011. Lisboa: INE; 2012.

16. Graffar M. Une méthode de classification sociale d'echantillons de population. Courier. 1956;6:4.

17. World Health Organization. Obesity: preventing and managing the global epidemic. Report of a WHO consultation. WHO Technical Report Series. 2000;894:1-253.

18. Santos O, Sermeus G, Carmo ID, Anelli M, Kupers P, Martin E. In search of weight loss - a four-country survey on what people were doing for losing weight at the turn of the century. Endocrinol Diabetes Obes. 2010;4:21-31.

19. Serdula MK, Williamson DF, Anda RF, Levy A, Heaton A, Byers T. Weight control practices in adults: results of a multistate telephone survey. Am J Public Health. 1994;84:1821-4. dos dados.

\section{CONFLITO DE INTERESSES}

Os autores declaram não haver conflito de interesses.

\section{FONTES DE FINANCIAMENTO}

Este estudo foi apoiado pela Fundação para a Ciência e a Tecnologia (SFRH/BD/80739/2011 atribuída a Inês Santos).

20. Serdula MK, Mokdad AH, Williamson DF, Galuska DA, Mendlein JM, Heath GW. Prevalence of attempting weight loss and strategies for controlling weight. JAMA. 1999;282:1353-8.

21. Villanueva EV. The validity of self-reported weight in US adults: a population based cross-sectional study. BMC Public Health. 2001;1:11.

22. Wooley SC, Garner DM. Obesity treatment: the high cost of false hope. J Am Diet Assoc. 1991;91:1248-51.

23. Johnson CC, Myers L, Webber LS, Hunter SM. Learned helplessness with excess weight and other cardiovascular risk factors in children. Am J Health Behav. 1997;21:51-59.

24. Bish CL, Blanck HM, Serdula MK, Marcus M, Kohl HW, Khan LK. Diet and physical activity behaviors among Americans trying to lose weight: 2000 Behavioral Risk Factor Surveillance System. Obes Res. 2005;13:596-607.

25. Yu Y. Educational differences in obesity in the United States: a close look at the trends. Obesity. 2012;20:904-8.

26. Roskam AJ, Kunst AE, Van Oyen H, Demarest S, Klumbiene J, Regidor $\mathrm{E}$, et al. Comparative appraisal of educational inequalities in overweight and obesity among adults in 19 European countries. Int J Epidemiol. 2010;39:392-404.

27. Marques-Vidal P, Paccaud F, Ravasco P. Ten-year trends in overweight and obesity in the adult Portuguese population, 1995 to 2005. BMC Public Health. 2011;11:772.

28. Direção-Geral da Saúde. Programa Nacional para a Promoção da Alimentação Saudável. Lisboa: DGS [consultado 2014 Abril 22]. Disponível em: http://www.alimentacaosaudavel.dgs.pt.

29. Timperio A, Cameron-Smith D, Burns C, Crawford D. The public's response to the obesity epidemic in Australia: weight concerns and weight control practices of men and women. Public Health Nutr. 2000;3:417-24.

30. Crawford D, Owen N, Broom D, Worcester M, Oliver G. Weight-contro practices of adults in a rural community. Aust $\mathrm{N} Z \mathrm{~J}$ Public Health. 1998;22:73-9.

31. Birch LL, Fisher JO, Davison KK. Learning to overeat: maternal use of restrictive feeding practices promotes girls' eating in the absence of hunger. Am J Clin Nutr. 2003;78:215-20.

32. Field AE, Manson JE, Laird N, Williamson DF, Willett WC, Colditz GA Weight cycling and the risk of developing type 2 diabetes among adult women in the United States. Obes Res. 2004;12:267-74.

33. Nicklas JM, Huskey KW, Davis RB, Wee CC. Successful weight loss among obese U.S. adults. Am J Prev Med. 2012;42:481-5.

34. Rodin J. Cultural and psychosocial determinants of weight concerns Ann Intern Med. 1993;119:643-5.

35. Tourangeau R, Yan T. Sensitive questions in surveys. Psychol Bull. 2007;133:859-83.

36. Merrill RM, Richardson JS. Validity of self-reported height, weight, and body mass index: findings from the National Health and Nutrition Examination Survey, 2001-2006. Prev Chronic Dis. 2009;6:A121.

37. Oliveira A, Ramos E, Lopes C, Barros H. Self-reporting weight and height: misclassification effect on the risk estimates for acute myocardial infarction. Eur J Public Health. 2009;19:548-53.

38. Yun S, Zhu BP, Black W, Brownson RC. A comparison of national estimates of obesity prevalence from the behavioral risk factor surveillance system and the National Health and Nutrition Examination Survey. Int J Obes. 2006;30:164-70.

39. Fitzgibbon ML, Stolley MR, Kirschenbaum DS. Obese people who seek treatment have different characteristics than those who do not seek treatment. Health Psychol. 1993;12:342-5. 


\section{Tentativas de Controlo do Peso na População Adulta Portuguesa: Prevalência, Motivos e Comportamentos}

Acta Med Port 2015:28:77-86

Publicado pela Acta Médica Portuguesa, a Revista Científica da Ordem dos Médicos

Av. Almirante Gago Coutinho, 151

1749-084 Lisboa, Portugal.

Tel: +351218428215

E-mail: submissao@actamedicaportuguesa.com

www.actamedicaportuguesa.com

ISSN:0870-399X | e-ISSN: 1646-0758

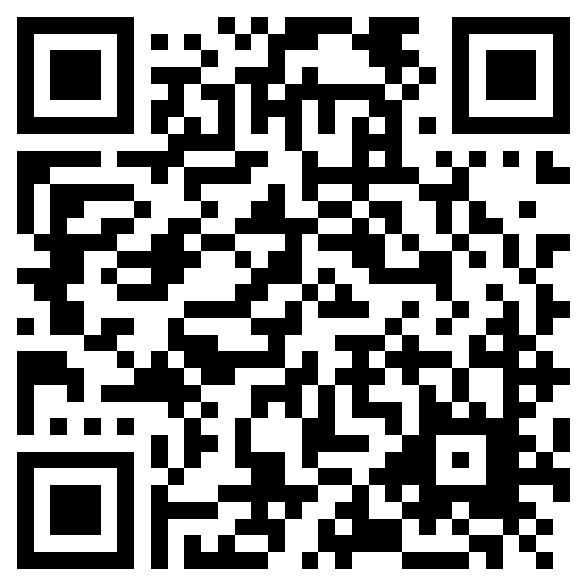

